\title{
Determinants of Numbers on Antenatal Care Visits in Rural Ethiopia: A Multilevel Negative Binomial Regression Analysis
}

Melkalem Mamuye Azanaw ( $\sim$ melkalem21@gmail.com )

Debre Tabor University

Alemayehu Digssie Gebremariam

Debre Tabor University

Fentaw Teshome Dagnew

Debre Tabor University

Hiwot Yisak Dawid

Debre Tabor University

Getaneh Atikilt Yemata

Debre Tabor University

Binyam Minuye Birhanie

Debre Tabor University

Melaku Tadege Engidaw

Debre Tabor University

Desalegn Tesfa Asnakew

Debre Tabor University

Edgiet Abebe Zewde

Debre Tabor University

Sofonyas Abebaw Tiruneh

Debre Tabor University

\section{Research Article}

Keywords: Antenatal care, Women, Rural Ethiopia, Multilevel Negative Binomial count analysis

Posted Date: March 12th, 2021

DOl: https://doi.org/10.21203/rs.3.rs-283138/v1

License: (c) (1) This work is licensed under a Creative Commons Attribution 4.0 International License.

Read Full License 
1 Determinants of numbers on Antenatal care visits in rural Ethiopia: A Multilevel negative

2 binomial regression analysis

Melkalem Mamuye Azanaw ${ }^{*}$, Alemayehu Digssie Gebremariam ${ }^{1}$, Fentaw Teshome Dagnew ${ }^{1}$, Hiwot Yisak Dawid ${ }^{1}$, Getaneh Atikilt Yemata ${ }^{1}$, Binyam Minuye Birhanie ${ }^{2}$, Melaku Tadege Engidaw $^{1}$, Desalegn Tesfa Asnakew ${ }^{1}$, Edgiet Abebe Zewde ${ }^{3}$, Sofonyas Abebaw Tiruneh ${ }^{1}$

Affiliation

${ }^{1}$ Department of Social and Public Health, College of Health Sciences, Debre Tabor University, Debre Tabor, Ethiopia, P.O. Box. 272.

${ }^{2}$ Department of Nursing, College of Health Sciences, Debre Tabor University, Debre Tabor, Ethiopia

${ }^{3}$ Department of Biomedical Sciences, College of Health Sciences, Debre Tabor University, Debre Tabor, Ethiopia

Email address

MMA:melkalem21@gmail.com;ADG:metsagu@yahoo.com; FTD:fentawtesh6@ gmail.com;

HYD: hyisak@yahoo.com;GAY: gechat9@gmail.com; BMB: biniamminuye@yahoo.com;

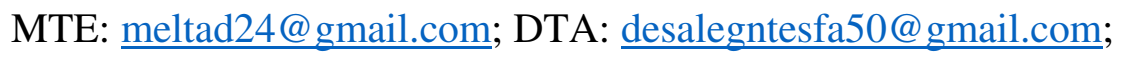

EAZ: edgetabebe82@gmail.com; SAT: zephab2@gmail.com

* Corresponding author

Melkalem Mamuye Azanaw

Melkalem21@gmail.com 


\section{Abstract}

Introduction: Antenatal care (ANC) is an umbrella term used to describe medical care and procedures that are carried out for pregnant women. Data on the number of antenatal care visits can help policymakers to show the gap in service provision. So, the paper determines associated factors of the number of antenatal care visits among women who gave birth in the last five years in rural Ethiopia.

Methods: Total weighted sample of 6611 women who gave birth in the 5 years preceding the survey were included from the nationally representative 2016 Ethiopian Demographic and Health Survey. We used multi-level negative binomial regression analysis to consider the hierarchal nature of the data. In the multivariable analysis, variables with a p-value $<0.05$ were considered to be significantly associated with the number of antenatal care visits.

Results: Overall, $27.3 \%$ (95\% CI: 14.63, 15.76) of women had at least four antenatal care visits during their pregnancy in rural Ethiopia. Age group 25-29 years (adjusted incidence rate ratio $(A I R R)=1.13,95 \%$ CI:1.02,1.26), household rich wealth status (AIRR=1.17, 95\% CI:1.04,1.31), women's educational status (primary, AIRR=1.19,95\% CI:1.08,1.32; Secondary, AIRR= 1.30,95\% CI:1.08,1.55; above secondary, AIRR=1.35, 95\%CI:1.07,1.71), partner educational status (primary, AIRR=1.16, 95\%CI:1.05,1.28; secondary, AIRR=1.22,95\% CI:1.08,1.38), and autonomy to decision to their care (AIRR=1.25,95\%CI:1.10,1.42) were positively associated individual-level factors with number of antenatal care visits whereas having a birth order of five or more $(A I R R=0.80,95 \%$ CI: $0.69,0.94)$ was a negatively associated factor with number of antenatal care visits. Among community-level variables; being in higher community level literacy $(\mathrm{AIRR}=1.35,95 \% \mathrm{CI}: 1.14,1.59)$ and higher poverty level (AIRR=0.77, 95\% CI: 0.64, 0.92) were significantly associated factors with the number of antenatal care visits.

Conclusions and recommendations: The level of antenatal care visits in rural areas was low. Age of women, wealth status, women's educational status, partner educational status, autonomy to decision making in health care, and birth order were the most important associated factors with the number of antenatal care visits. Furthermore, poverty and literacy are also important determinants at the community level. Addressing economic and educational interventions for rural women should be given the top priority.

2 Keywords: Antenatal care, Women, Rural Ethiopia, Multilevel Negative Binomial count analysis 
35 Antenatal care (ANC) is an umbrella term that describes medical care and procedures that are 36 carried out for pregnant women (1). It is the health care delivery that is given to pregnant women 37 throughout their pregnancy. Services during ANC visit aimed at detecting the already existing 38 problems and/or problems that might be developed during pregnancy, which may affect the 39 pregnant woman and/or her unborn child (2). Screening tests, diagnostic procedures, and 40 prophylactic treatments are the services provided to pregnant women based on identified problems 41 and risk factors (3).

42 The World Health Organization (WHO) recommends all pregnant women and newborns get 43 quality care. Recommended activities to assure the quality of care are; nutritional interventions, 44 maternal and fetal assessment, preventive measures, interventions for common physiological 45 conditions, and health systems interventions (4). By implementing timely and proper evidence46 based practice, ANC can save lives. Remarkably, ANC also helps to communicate and help 47 women, families, and communities in a dangerous situation in a woman's life (5).

48 Antenatal care services utilization is one of the most important health services in combating 49 morbidity and mortality among pregnant mothers and the utilization of appropriate and 50 recommended antenatal care services supports safe maternity and delivery which finally improved 51 maternal and child health (6).

52 Many efforts have been done to assess the evolution in coverage of antenatal care in developing 53 countries which focused on issues like the number of visits, the timing of visits, and the 54 characteristics of users and non-users of antenatal care (7). 
55 The number of antenatal care services is determined by socio-demographic characteristics and 56 contextual causes. Regarding individual factors, higher educational status, older age, and higher57 income were positively associated with the number of antenatal care visits. Fewer antenatal care 58 visits were associated with parity, gestational age at delivery, time of initiation of ANC, and 59 medical risks during the pregnancy. Concerning contextual factors; distance from a health facility, 60 service waiting time, and client welcomed appreciation were associated with ANC services 61 utilization. Besides, women's knowledge regarding ANC, the presence of electricity in 62 households, and housing conditions were associated with ANC services utilization $(8,9,18-$ $6327,10,28-32,11-17)$.

64 Even though antenatal care is crucial, the information in rural areas of Ethiopia is still very limited 65 due to different factors. Little is known about factors affecting the number of antenatal care visits 66 in rural Ethiopia. Thus, a detailed investigation of the factors affecting the number of ANC service 67 utilization is crucial. That is why this study was conducted to analyze the number of ANC visits 68 and determinants among women who gave birth in the last five years preceding the survey in rural 69 Ethiopia. Besides, this study valuable information on count data models. 
Methods

\section{Study area and data source}

78 The study was conducted in Ethiopia, which is located in the horn of Africa, lies between $3^{0}$ and $7915^{0}$ North latitudes and $33^{\circ}$ and $48^{0}$ East longitudes. We used the EDHS 2016 dataset which was conducted by the Central Statistical Agency (33) in collaboration with the Federal Ministry of

81 Health $(\mathrm{FMoH})$ and the Ethiopian Public Health Institute (EPHI). The Ethiopian DHS is conducted 82 every 5 years to assess population and health-related indicators of the country. Data were obtained

83 from the DHS website (URL: www.dhsprogram.com) after contacting them via email through 84 personal accounts and justifying the reason for requesting it.

\section{Population and sampling procedure}

86 The study was conducted using nationally representative data from the 2016 Ethiopian

87 Demographic and Health Survey. The current study used 443 EAs from 645 EDHS EAs or clusters

88 as identified by the 2007 Ethiopia Population census. A stratified two-stage sampling procedure

89 was employed to identify households from all eligible rural households within each cluster The source population were all women of reproductive age who gave birth within five years before the

91 survey in rural Ethiopia. The study population included women of reproductive age who gave birth

92 within five years before the survey in the households located in the primary sampling units (PSUs)

93 that are in the 443 Enumeration areas sampled in the first stage.

\section{$94 \quad$ Variables}

95 The outcome variable was the number of ANC visits each participant had in the last pregnancy.

96 Individual (woman), household, community, and regional level variables have been considered as

97 explanatory variables. The individual-level explanatory variables included education status of the 
women and their husbands, age of the women, the autonomy of the women to healthcare issues, women's and husbands' occupational status, birth order of a child, and household wealth status whereas community-level variables were regional settings, community-level poverty, and literacy.

\section{Data processing and analysis}

The data were cleaned using STATA version 16.0 software. The analysis was conducted after sample weights were applied for complex sampling procedures. The characteristics of the study participants were described using frequencies and percentages.

Let $\mathrm{Y}_{\mathrm{ij}}$ represent the number of ANC visits of the $\mathrm{i}^{\text {th }}$ women living in the $\mathrm{j}^{\text {th }}$ cluster, and the vector $\mathrm{X}_{\mathrm{ij}}$ the corresponding values of the independent variables. Assuming independent women who are on ANC visits, Poisson regression and negative binomial regression models are specified as:

$$
\log \left(\mu_{i j}\right)=\beta_{0}+\beta X_{i j}
$$

Where $\mu_{i j}$ is the expected number of ANC visits as a function of independent variables, $\beta_{0}$ the overall intercept, and $\beta$ the vector of regression coefficients. The assumed distribution of number of ANC visits are the difference between the Poisson regression (PR) and the negative binomial regression model (NBR). In PR, the outcome variable is assumed to follow a Poisson distribution with $E\left(y_{i j}\right)=\mu_{i j}=\operatorname{var}\left(y_{i j}\right)$, while in NBR, it is assumed to follow a negative binomial distribution with $\mathrm{E}\left(y_{i j}\right)=\mu_{i j}$ and $\operatorname{var}\left(y_{i j}\right)=\theta \mu_{i j}$, where $\theta$ is the shape parameter that controls the variance. When $\theta=1$, the NBR model becomes PR model without over-dispersion.

When the assumptions of independence are violated in clustered data, using PR and NBR models could lead to biased estimates and misinterpretation of the results (34). The best way to consider the non-independence of observations is to use multilevel models. The use of a multilevel modeling strategy accommodates the clustered or hierarchical nature of the EDHS data and adjusts 
120 standard errors of the estimated coefficients for ICC. A simple multilevel PR/NBR model is

121 obtained by incorporating cluster-specific random effects in the standard PR/NBR model:

$$
\log \left(\mu_{I J}\right)=\left(\beta_{0}+b_{0 j}+\beta X_{i j}\right.
$$

123 Where $b_{0 j}$ denotes the random intercepts at cluster level and are assumed to follow a normal 124 distribution with constant variance. Therefore, the multilevel PR and NBR models are preferred to 125 model the data(35).

126 The random effects (variation of effects) were estimated by intracluster correlation coefficient 127 (ICC) (variance partition coefficient) and the percentage change in variance (PCV). The ICC 128 explains the cluster variability whereas PCV measures the total variation due to factors at the 129 community and individual levels. The ICC and PCV were determined using the following 130 formulas:

$$
I C C=\text { the variance of the null model/(variance of the null model }+3.29)
$$

$$
P C V=\frac{\text { (variance of the initial model }- \text { variance of the model with more terms) }}{\text { the variance of the initial model }} * 100
$$

134 A multilevel model provides correct parameter estimates by adjusting the biases introduced from 135 clustering to give correct SEs. Thus, it produces correct confidence interval and significance 136 tests(36).

137 The goodness of fit statistics by Pearson Chi-square of Poisson regression analysis was conducted 138 to show whether over-dispersion is present or not. If the observed value of the Pearson Chi-square 139 statistic divided by the degrees of freedom is higher than one, the mentioned goodness of statistics 140 represents over-dispersion in the data set. 


\section{Operational definitions}

143 Number of Antenatal care visit: Is the number of health care visit of women who gave birth five

144 years preceding the survey, information was recorded for the last birth.

145 Community women's literacy: categorized into two as a higher proportion of women's literacy

146 within the cluster and a lower proportion of women's literacy based on the median value. The

147 aggregate of individual women's ability to read-only parts of a sentence, able to read the whole

148 sentence, and no card with required language can show the overall literacy status of women within

149 the cluster.

150 Community-level poverty: categorized into two as a higher proportion of poorest and poorer

151 households within the cluster and lower poorest and poorer households based on the median value.

152 The aggregate of individual women from the poorest and poorer households can show the overall

153 community-level poverty of women within the cluster.

154

155

156

157

158

159

160

161 


\section{Results}

\section{Socio-demographic characteristics of respondents}

165 The total number of women was 6611 with mean $( \pm \mathrm{SD})$ ages of $29.7( \pm 7.4)$ years. About 3,219 $166(48.7 \%)$ mothers were in the age of 30-45 years. Approximately $70 \%$ of women and were non167 educated with 224(3.4\%) secondary and above the secondary school. Almost all (>94\%) of women 168 were married. About 2,916 (44.1\%) of women were from the Oromia region and 2,596(39.3\%) 169 women were Muslim religion followers. Moreover, 3,681(55.7\%) of the women had no work. 170 Regarding wealth status, nearly half (49.1\%) of the women were from poor or poorest households 171 whereas, $1,803(27.2 \%)$ belonged to rich and richest households during the five years preceding the 172 survey (Table 1).

\section{Community-level, maternal, and health service-related characteristics}

174 Approximately $74.0 \%$ of women had autonomy for the decision of health care, $4,240(64.1 \%)$ of 175 them had big problems in distance to the health facility. Regarding birth order, 2,037 (30.8\%) 176 women had a child with above five birth order whereas, 1,099(16.6\%) had first birth order child 177 (Table 2).

\section{Number of ANC visits during pregnancy}

179 The median and mode of the frequency of antenatal care visits were 2 and 0 among mothers who 180 gave birth within the last five years respectively. Overall, 58.8\% (95\%CI: 57.6, 59.9) of women

181 had at least one ANC visit during their pregnancy and only $27.3 \%$ (95\% CI: $14.63,15.76)$ of 182 women had at least four times ANC visits during their pregnancy in rural Ethiopia (Fig1). 


\section{Factors associated with Number of Antenatal care Visits}

About $10.7 \%$ of the number of antenatal care visits during pregnancy in clusters was explained in the null model. The Percent change variation in the final model showed that about $74.9 \%$ of the total variability in the number of antenatal care visits was explained (Table3).

The multilevel negative binomial regression model analysis of individual factors that had a significant association with the number of antenatal care visits was age, educational status of women, educational status of the husband, occupational status of the women, household wealth, women's decision-making power on their healthcare issues, and birth order of child whereas, community-level literacy, and poverty determine the number of antenatal care visits (Table 4).

Women with the age range of 25 to 29 years had $13 \%$ more antenatal visits compared with women age 15-24 years (IRR: 1.13, 95\% CI: 1.02-1.26). Women who were attending primary, secondary, and above secondary education had more antenatal care visits compared with women who had no educational status (primary education; IRR: 1.16, (95\% CI: 1.05, 1.28), secondary IRR: 1.22, (95\% CI: 1.08, 1.38), and above IRR: 1.35, (95\% CI: 1.07, 1.71)). Partner with primary education and secondary had $16 \%$ and $22 \%$ more antenatal visits compared with a partner who had no educational status (IRR: 1.16, (95\% CI: 1.05, 1.28) and IRR: 1.22, (95\% CI: 1.08, 1.38), respectively). Women from richer households had $17 \%$ more antenatal care visits compared with women from poorer households (IRR: 1.17, (95\% CI: 1.04, 1.31). Women who had the decision-making power to their health care were $25 \%$ more antenatal care visits (IRR: 1.25 , (95\% CI: $1.10,1.42)$. On the other hand, women who had a higher birth order child had lower antenatal care visits compared to women who had first order child (IRR: 0.80, (95\%CI: 0.69, 0.94).

Furthermore, community-level variables were significantly associated with the number of antenatal care visits after controlling the possible confounding by multivariate analysis. Women 
208 from higher literacy level communities had 35\% higher antenatal care visits (IRR: 1.35, (95\%CI:

$2091.14,1.59)$ whereas women from the higher poverty level community had $23 \%$ lower antenatal 210 care visits (IRR: 0.77 (95\%CI: 0.64, 0.92) (Table3).

211

212

213

214

215

216

217

218

219

220

221

222

223

224

225

226

227

228

229

230 


\section{Discussion}

232 Antenatal care is an indicator to measure the efficiency of maternal care utilization. It helps in 233 preventing adverse pregnancy outcomes when provided early in the pregnancy and continued 234 through delivery. Identification of problems in pregnancy results in early referrals for women with 235 complications; this is particularly true in Ethiopia, where three-quarters of the population live in 236 rural areas. So as this study focused on determinants of the number of Antenatal care visits in rural 237 Ethiopia using the multilevel negative binomial analysis to estimate individual, household, 238 community-level factors.

239 The current study estimated that $27.3 \%$ of pregnant women in rural Ethiopia have been received 240 at least four ANC during their pregnancy. It showed an improvement as compared to the 2011 data 241 which was a $47 \%$ increase(37). This increment might be due to awareness creation activities, health 242 promotion, and an increase in the number of health institutions and health care providers in rural $243 \operatorname{areas}(38)$.

244 In this study, individual-level and community-level factors were responsible for around $75 \%$ of 245 the difference in the frequency of ANC visits during pregnancy in rural Ethiopia. After adjusting 246 all factors, the frequency of ANC visits was higher among those pregnant mothers in the age range 247 of 25 to 29 years, higher educational status of women and their partner, highest wealth quintile, 248 women who had the autonomy to decide their health care, and community level literacy. However, 249 women who had a higher birth order child and women from low socioeconomic status of the 250 community had a lower frequency of ANC visits.

251 The current study showed that women who were in the age group of 25-29 years were 13 252 percentage points more likely to visit health institutions for ANC services compared with those 253 who were in the age group of 15-24 years. This finding supports previous studies done in different 
254 countries $(6,39,40)$ that showed the positive association between ANC visits and increased age of

255 women. This might be due to health conditions and birth complications are higher in older women

256 who tend to demand more visits. But it is not in line with studies done in metropolitan

257 countries(10).

258 The finding of the study also indicated that women who had primary, secondary, and above 259 secondary educational attainment were 19, 30, and 35 percentage points more likely to use ANC 260 services as compared with those who had no education respectively. This finding is consistent with 261 the study conducted in Ghana(6,31). The statistical relationship between the utilization of maternal 262 health services and education is also well documented in a systematic review of 74 studies and 263 Bangladesh(22,34). This indicates that the educational attainment of the women increases 264 awareness of the need to access health services by frequent antenatal care visits. The primary and 265 secondary educational status of the husband was also more likely to have a high frequency of ANC 266 visits as compared to the non-educated husband. This finding supports that the high educational 267 attainment of the husband influenced the frequency of ANC visits in sub-Saharan countries(22,34). 268 This implied that educated partners may be more concerned with their pregnant wives and 269 associated pregnancy complications.

270 Furthermore, the multilevel negative binomial estimation indicates that the wealth status of the 271 household had a positive and significant effect on the frequency of ANC visits. The result showed 272 that pregnant women in the richer household were more likely to have frequent ANC visits relative 273 to those in the poorest household. This finding agreed with a study conducted in different countries 274 in the world $(6,8,34)$. But, the positive association does not vary by urban-rural settings in these 275 studies. Thus, the results suggested household wealth status could be an important variable for 276 antenatal care utilization. This might be since having a low economic status would mean having 
277 less money for transportation towards the health facility for utilization of ANC service. Another

278 possible explanation could be women who belong to rich families usually have proper education,

279 access to mass media than from poorer families.

280 Besides, the presence of women's autonomy in their health care was related to more antenatal care

281 visits. This indicates the importance of women's empowerment, not only within the household but

282 also in the community. Women empowerment leads to greater decision-making power concerning

283 maternal health. Our findings are consistent with a previous research study conducted in

284 Bangladesh(34), which revealed that women who decided about their medical care were more

285 likely to receive professional antenatal care.

286 The current finding also showed that the birth order of the child influences the number of antenatal

287 care visits. Having higher birth order child was negatively and significantly associated with the

288 number of ANC visits. This finding agreed with a study done in Ghana(6). This implies that

289 mothers with more previous birth experiences demand lesser visits, all other things being equal.

290 From community-level variables, literacy at the community level was a significant factor that

291 determined the number of ANC visits. A high frequency of antenatal care visits was associated

292 with literacy in a rural population. This result is in line with the study done in India(8).

\section{Strengths and limitations}

294 We used large population-based data with a large sample size, which is representative of all rural 295 regions of Ethiopia. Furthermore, a count statistical analysis (multilevel Negative Binomial 296 regression analysis) was applied for this study that allows the effects of each determinant on the 297 frequency of ANC services efficiently. The novelty of this paper lies in the fact that we have 298 modeled the determinants of the number of antenatal care services in rural Ethiopia using the most 299 recent DHS. One significant point of departure of this study from previous studies on Ethiopia is 
300 the inclusion of only rural place of residence. Even though, the cross-sectional nature of the EDHS

301 data, reports of this finding explained by incidence rate ratio. The temporal relationship could not

302 be established based on these study findings. Ethiopian demographic and health surveys were

303 questionnaire-based surveys and depend on the memory of the respondents, and as such, recall

304 bias in the results might be a limitation for this study.

305 Conclusion and recommendations

306 The level of antenatal care visits among women who gave birth within the last five years before

307 the survey was low in rural Ethiopia. Our results also indicate that wealth status, age, women and

308 partner educational status, the decision-making power of women to their health care, community-

309 level poverty, and literacy are significant factors affecting the number of antenatal care in rural

310 Ethiopia. Besides, birth order is inversely and significantly associated with the frequency of ANC

311 visits. The findings of this report help policymakers and programmers to focus their programs and

312 plan in rural parts of the country to enhance maternally and child health. Priority should be given

313 to women and their partners of low educational status to get appropriate maternal and child health

314 care service promotions. However, ANC service is provided for free, interventions have to be in

315 place to improve the household wealth status since household wealth status still plays a major role

316 in the frequency of ANC follow-up. 


\section{Abbreviations}

324 WHO: World Health Organization; ANC: antenatal care services; IRR: incidence rate ratio;

325 EDHS: Ethiopia Demographic and Health Survey; CI: confidence interval; NB: negative binomial;

326 PR: Poisson regression

327 Authors' contributions

328 MMA, ADG, FTD, DTA, and SAT made substantial contributions to the conception and design 329 of the study, the formulation of the methodology, analyzed, interpreted the data, and reviewed the 330 final version submitted for publication. HYD, GAY, BMB, MTE, and EAZ reviewed the final 331 version submitted for publication. All authors read and approved the final manuscript.

332 Acknowledgments

333 Our deepest gratitude goes to the Central statistical Agency for providing the data.

334 Competing interests

335 The authors declare that they have no competing interests.

336 Availability of data and materials

337 The datasets used and/or analyzed during the current study available from the corresponding author 338 on reasonable request.

339 Consent for publication

340 Not applicable as there are no image or other confidentiality-related issues.

341 Ethics approval and consent to participate

342 It is not applicable, because the data was collected by the performance monitoring and 343 accountability 2020 (PMA2020)/Ethiopia survey project.

$344 \quad$ Funding

345 Not applicable 


\section{Reference}

347 1. Ekabua J, Ekabua K, Njoku C. Proposed Framework for Making Focused Antenatal Care

Services Accessible: A Review of the Nigerian Setting. ISRN Obstet Gynecol. 2011;2011(December):1-5.

2. Sylvia Patience Ngxongo T. Basic Antenatal Care Approach to Antenatal Care Service Provision. Sel Top Midwifery Care. 2019;1-14.

352 3. Picco S, Villegas L, Tonelli F, Merlo M, Rigau J, Diaz D, et al. Basic Antenatal Care Approach to Antenatal Care Service Provision. Web Sci. 2016;(tourism):13.

354 4. Health R, Health A. WHO Guideline on Antenatal Care ( 2016 ). Dev Guidel. 2016;1-46.

355 5. Otundo Richard M. WHO Recommendations on Antenatal Care for a Positive Pregnancy $356 \quad$ Experience in Kenya. SSRN Electron J. 2019;7(2):1-16.

357 6. Nketiah-Amponsah E, Senadza B, Arthur E. Determinants of the utilization of antenatal 358 care services in developing countries: Recent evidence from Ghana. African J Econ Manag 359 Stud. 2013;4(1):58-73.

360 7. CL A-Z, T W. Antenatal Care in Developing Countries. Promises, Achievements and 361 Missed Opportunities: An Analysis of Trends, Levels and Differentials, 1990-2001. WHO Library Cataloguing-in-Publication Data Antenatal. 2003. 1-32 p.

363 8. Chandhiok N, Dhillon BS, Kambo I, Saxena NC. Determinants of antenatal care utilization 364 in rural areas of India : A cross-sectional study from 28 districts ( An ICMR task force study ). J Obstet Gynecol India. 2006;56(1):47-52.

366 9. Tovar LM. Individual and local level factors and antenatal care use in Colombia: a multilevel analysis. Cad Saúde Pública,. 2014;30(5):1079-92.

368 10. Beeckman K, Louckx F, Putman K. Determinants of the number of antenatal visits in a 
metropolitan region. BMC Public Health. 2010;10:1-9.

370 11. Manyeh AK, Amu A, Williams J, Gyapong M. Factors associated with the timing of antenatal clinic attendance among first-time mothers in rural southern Ghana. BMC Pregnancy Childbirth. 2020;20(1):1-7.

373 12. Shibre G, Zegeye B, Idriss-Wheeler D, Ahinkorah BO, Oladimeji O, Yaya S. 374 375 376 377

15. Ergat Yarinbab T. Determinants of Antenatal Care Service Utilization during the First Trimester among Pregnant Women in Boke Woreda, Ethiopia: A Facility Based Unmatched Case-Control Study. Curr Trends Biomed Eng Biosci. 2018;15(1):13-20.

16. Tusa BS, Weldesenbet AB, Kebede SA. Spatial distribution and associated factors of underweight in Ethiopia: An analysis of Ethiopian demographic and health survey, 2016. PLoS One. 2020;15(12 December).

17. Gebremeskel F, Dibaba Y, Admassu B. Timing of First Antenatal Care Attendance and Associated Factors among Pregnant Women in Arba Minch Town and Arba Minch District, Gamo Gofa Zone, South Ethiopia. J Environ Public Health. 2015;2015. 
392 18. Kistiana S. Socio-Economic and Demographic Determinants of Maternal Health Care Utilization in Indonesia. Environ Manage. 2009;2(2):212-9.

394 19. Neupane S, Doku DT. Determinants of time of the start of prenatal care and number of 395 prenatal care visits during pregnancy among Nepalese women. J Community Health. 396 2012;37(4):865-73.

397 20. Kyei NNA, Campbell OMR, Gabrysch S. The Influence of Distance and Level of Service 398 Provision on Antenatal Care Use in Rural Zambia. PLoS One. 2012;7(10).

399 21. Manithip C, Sihavong A, Edin K, Wahlstrom R, Wessel H. Factors associated with antenatal 400 care utilization among rural women in Lao people's democratic republic. Matern Child 401 Health J. 2011;15(8):1356-62.

402 22. Okedo-Alex IN, Akamike IC, Ezeanosike OB, Uneke CJ. Determinants of antenatal care 403 utilization in sub-Saharan Africa: A systematic review. BMJ Open. 2019;9(10):1-14.

404 23. Teshome MS, Meskel DH, Wondafrash B. Determinants of anemia among pregnant women 405 attending antenatal care clinic at public health facilities in kacha birra district, southern 406 Ethiopia. J Multidiscip Healthc. 2020;13:1007-15.

407
24. Amin R, Shah NM, Becker S. Socioeconomic factors differentiating maternal and child health-seeking behavior in rural Bangladesh: A cross-sectional analysis. Int J Equity Health. 2010;9:1-11.

25. Adamu YM, Salihu HM. Barriers to the use of antenatal and obstetric care services in rural Kano, Nigeria. J Obstet Gynaecol (Lahore). 2002;22(6):600-3.

26. Petrou S, Kupek E, Vause S, Maresh M. Clinical, provider and sociodemographic determinants of the number of antenatal visits in England and Wales. Soc Sci Med 52. 2001;52:1123-34. 
415 27. Islam MM, Masud MS. Determinants of frequency and contents of antenatal care visits in 416 Bangladesh: Assessing the extent of compliance with the WHO recommendations. PLoS 417 One. 2018;13(9):1-22.

418 28. Metgud CS, Katti SM, Mallapur AS, Wantamutte. Utilization Patterns of Antenatal Services 419 420

421

29. Visits A. Determinants of the number of Antenatal Visits in Somalia §. 2018;(22).

422 30. Ousman SK, Mdala I, Thorsen VC, Sundby J, Magnus JH. Social determinants of antenatal 423 care service use in Ethiopia: Changes over 15 years. Front Public Heal. 2019;7(JUN):1-10.

424 31. Overbosch GB, Nsowah-Nuamah NNN, van den Boom GJM, Damnyag L. Determinants of 425 antenatal care use in Ghana. J Afr Econ. 2004;13(2):277-301.

426 32. Rurangirwa AA, Mogren I, Nyirazinyoye L, Ntaganira J, Krantz G. Determinants of poor 427 utilization of antenatal care services among recently delivered women in Rwanda; a 428 population-based study. BMC Pregnancy Childbirth. 2017;17(1):1-10.

429 33. 2016 CSA (CSA) [Ethiopia] and ICF. EDHS 2016. Addis Ababa, Ethiopia, and Rockville, 430 Maryland, USA: CSA and ICF. 2017.

431 34. Bhowmik KR, Das S, Islam MA. Modeling the number of antenatal care visits in 432 Bangladesh to determine the risk factors for reduced antenatal care attendance. PLoS One. 2020;15(1):1-17.

$434 \quad$ 35. Stata. Menbreg. 2014;

435 36. Merlo J, Chaix B, Ohlsson H, Beckman A, Johnell K, Hjerpe P, et al. A brief conceptual 436 tutorial of multilevel analysis in social epidemiology: Using measures of clustering in multilevel logistic regression to investigate contextual phenomena. J Epidemiol Community 
Health. 2006;60(4):290-7.

439 37. Agency CS, Ababa A. Ethiopia Demographic and Health Survey. 2012;(March).

440 38. Workie MS, Lakew AM. Bayesian count regression analysis for determinants of antenatal care service visits among pregnant women in Amhara regional state, Ethiopia. J Big Data.

442 2018;5(1).

443 39. Kupek E, Petrou S, Vause S, Maresh M. Clinical, provider and sociodemographic predictors of late initiation of antenatal care in England and Wales. BJOG An Int J Obstet Gynaecol. 2002;109(3):265-73.

446 40. Hildingsson I, Råoestad I, Waldenström U. Number of antenatal visits and women's opinion. Acta Obstet Gynecol Scand. 2005;84(3):248-54.

448

449 
Figures

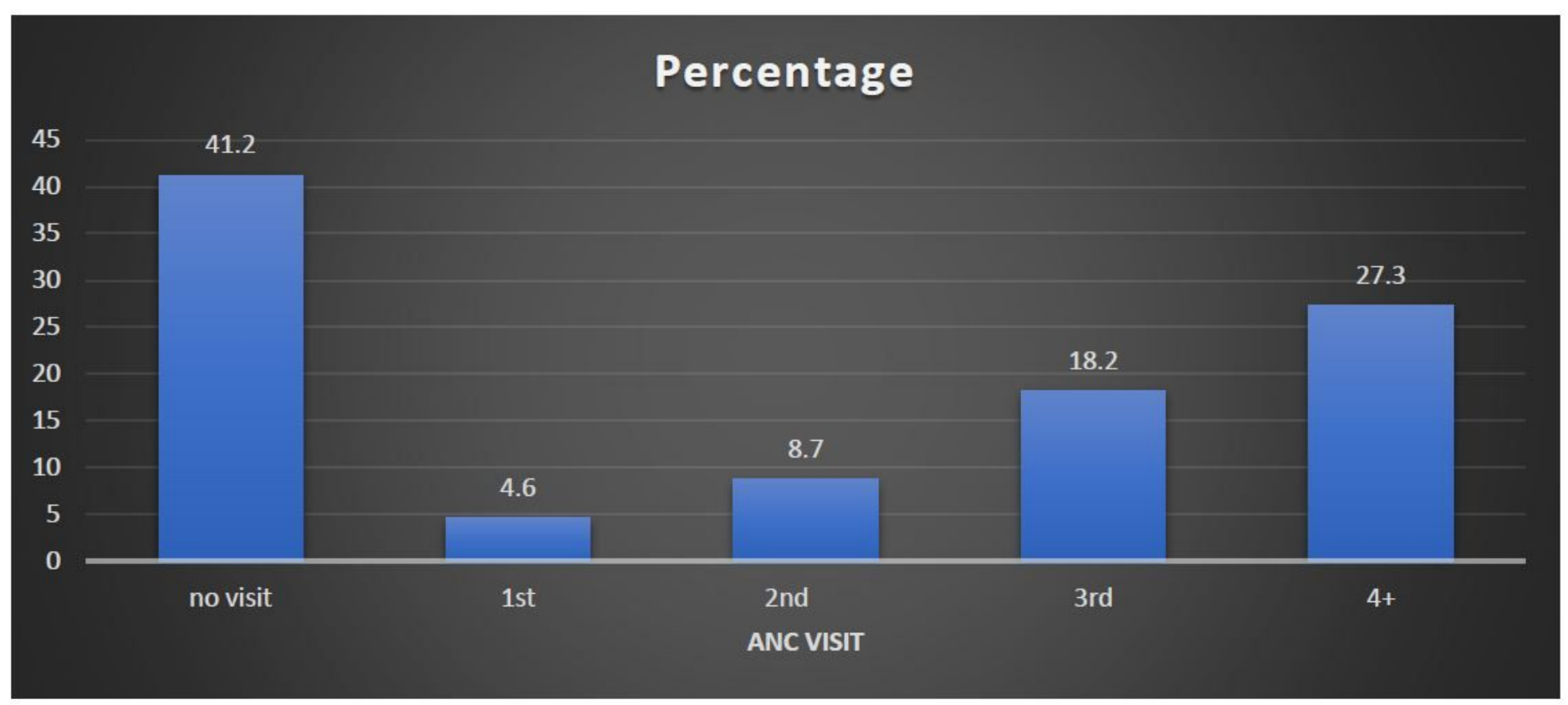

Figure 1

proportion of the number of antenatal care visits for women who gave birth within the last five years preceding the survey in rural Ethiopia, EDHS 2016, 2020( $\mathrm{N}=6611)$. 